\title{
Acute Common Carotid Artery Bifurcation Thrombus: An Emerging Pattern of Acute Strokes in Patients with COVID-19?
}

W e read with great interest the recent article by Goldberg et al $^{1}$ entitled, "Cerebrovascular Disease in COVID-19.” In a patient with coronavirus 2019 (COVID19) illness, the authors presented a case of evolving acute infarcts on CT head examinations at different time points, as well as high-grade stenosis of the proximal right internal carotid artery. With regard to the right internal carotid artery, the authors state, "Given that the patient had evidence of mild, diffuse extracranial and intracranial atherosclerotic disease, the high-grade stenosis of the right ICA was favored to represent atherosclerotic disease; however, acute thrombus could have a similar appearance." While the authors may be correct in their interpretation of the case, we suspect that carotid thrombosis is a very frequent sequela of COVID-19-associated stroke. We have recently seen cases of acute stroke, in the setting of COVID-19 infection, with imaging features consistent with acute thrombosis in the ipsilateral common carotid artery bifurcation, rather than progression of atherosclerotic disease.

For example, a 53-year-old woman presented to our institution with increasing confusion, following recent discharge from another institution, at which COVID-19-related stroke was diagnosed. MR imaging of the brain performed at the outside institution demonstrated acute-subacute infarcts in the right frontal and parietal lobes (not shown). CTA of the head and neck performed at our institution demonstrated an elongated, eccentric filling defect spanning a 1-cm segment of the proximal right internal carotid artery, consistent with intraluminal thrombus (Fig 1). Notably, there was no other evidence of intracranial or extracranial atherosclerotic disease. In a second case, a 49-year-old man presented with acute aphasia and right-sided weakness. CTA of head and neck demonstrated a left middle cerebral artery M1 occlusion (not shown). Eccentric filling defects were present along the posterior aspect of the left common carotid artery bifurcation extending into the

- Indicates open access to non-subscribers at www.ajnr.org

http://dx.doi.org/10.3174/ajnr.A6657 left internal carotid artery, compatible with thrombus (Fig 2). As in the first case, there was no other evidence of intracranial or extracranial atherosclerotic disease. The patient tested positive for COVID-19.

In both cases, the imaging findings at the proximal internal carotid arteries were suggestive of acute thrombus: elongated and eccentric filling defects extending into the lumen without other signs of intra- or extracranial atherosclerotic disease. Consequently, these cases highlight patients with COVID-19 presenting with acute strokes likely related to ipsilateral common carotid artery bifurcation acute thrombus.

Growing evidence suggests that COVID-19 is associated with acute cerebrovascular disease, often in patients who would otherwise be at low risk for stroke. ${ }^{2}$ As noted by Goldberg et al, "Systemic infection . . . is associated with activation of immune and inflammatory pathways leading to plaque disruption, serving as a source of thrombosis." COVID-19 infection may predispose patients to thrombotic disease involving either the arterial or venous circulations, secondary to increased inflammation, platelet activation, endothelial dysfunction, and stasis. ${ }^{3}$ A recent case report by Singh et $\mathrm{al}^{4}$ described an urgent carotid endarterectomy in a patient with COVID-19 with right-sided acute infarcts and right internal carotid artery stenosis, with duplex sonography demonstrating $85 \%$ stenosis. During the operation, perivascular inflammation was noted, and a soft friable plaque was present on opening the vessel. On histopathology, the plaque demonstrated blood, fibrin, and acute and chronic inflammation.

Our experience supports other observations of patients with COVID-19 presenting with acute strokes secondary to acute thrombus within the ipsilateral common carotid artery bifurcation, possibly as the result of postinfectious inflammatory thrombosis formation. We thank Goldberg et $\mathrm{al}^{1}$ for their important case description. We believe that investigational studies are needed to evaluate acute thrombus formation at the common carotid artery bifurcation as a potential cause of acute strokes in patients with COVID-19. 


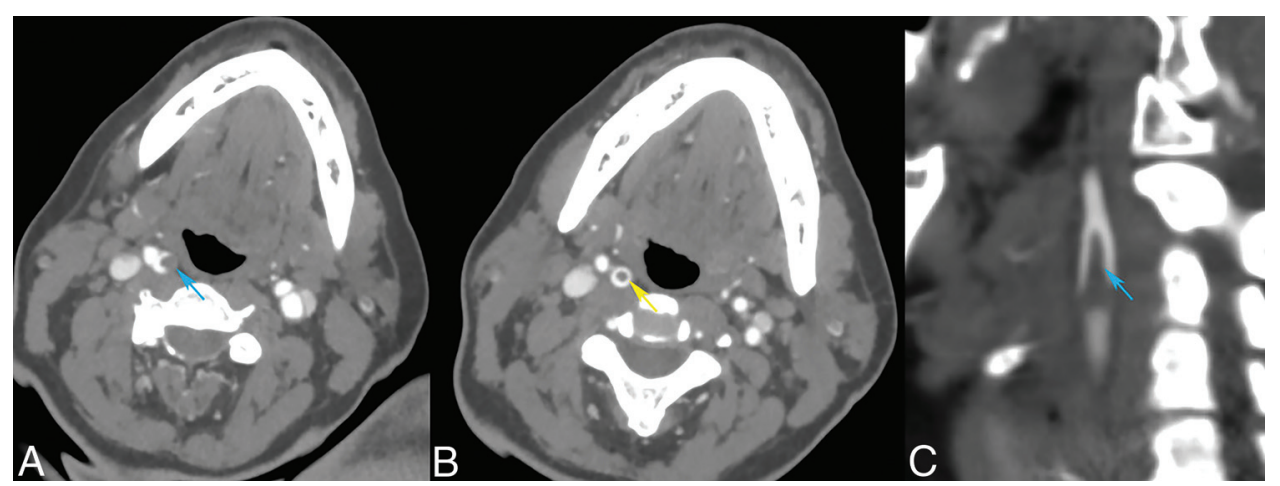

FIG 1. Axial $(A$ and $B)$ and sagittal MIP $(C)$ images from a CT angiogram in a patient with COVID-19 with right frontal and parietal acute-subacute infarcts. There are eccentric filling defects within the right common carotid artery bifurcation extending into the right internal carotid artery (blue arrows), with creation of a donut sign (yellow arrow). Atherosclerotic changes were not present in the remainder of the intracranial and extracranial arterial vasculature. Findings are suggestive of acute thrombus.

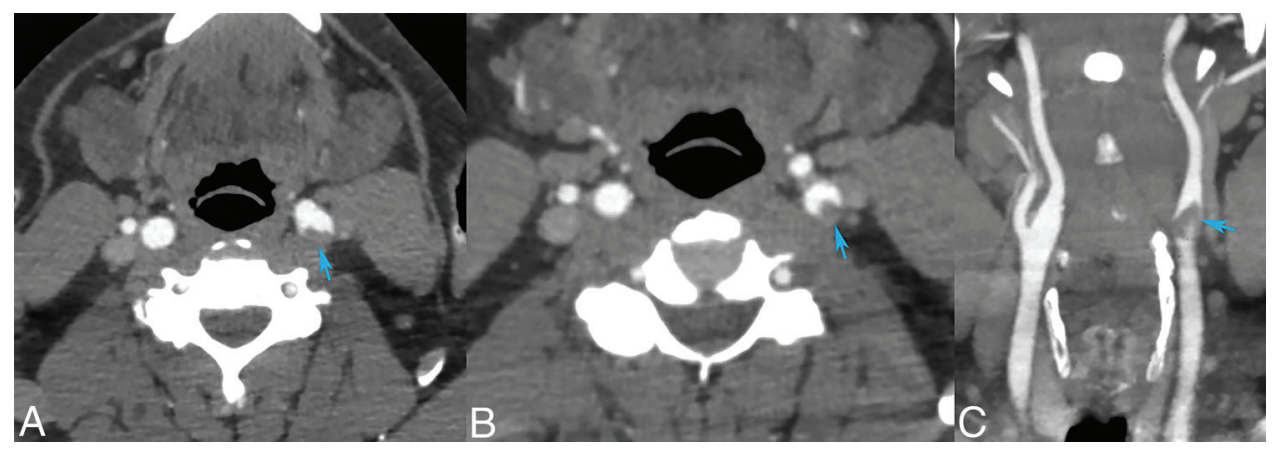

FIG 2. Axial $(A$ and $B)$ and coronal $(C)$ images from a $C T$ angiogram in a patient presenting with left MCA syndrome and a left middle cerebral artery $\mathrm{M} 1$ occlusion. There are eccentric filling defects within the left common carotid artery bifurcation extending into the left internal carotid artery. There are eccentric filling defects within the left common carotid artery bifurcation extending into the left internal carotid artery (blue arrows). Findings are suggestive of acute thrombus.

\section{REFERENCES}

1. Goldberg MF, Goldberg MF Cerejo R, et al. Cerebrovascular disease in COVID-19. AJNR Am J Neuroradiol 2020;41:1170-72 CrossRef (1)W. Gomes Medline

2. Oxley TJ, Mocco J, Majidi S, et al. Large-vessel stroke as a presenting feature of Covid-19 in the young. N Engl J Med 2020;382:e60 CrossRef Medline

3. Bikdeli B, Madhavan MV, Jimenez D, et al. COVID-19 and thrombotic or thromboembolic disease: implications for prevention, antithrombotic therapy, and follow-up. J Am Coll Cardiol 2020 Apr 15. [Epub ahead of Print] CrossRef Medline

4. Singh T, Lee A, Vo R, et al. Urgent carotid endarterectomy in a COVID-19 patient: standard approach with some adjustments. Vascular Disease Management 2020;17:E104-09 